\title{
NON DIFFUSIVE PROPAGATION OF SOLAR ENERGETIC PARTICLES: DATA ANALYSIS AND NUMERICAL SIMULATIONS
}

\author{
E.M. Trotta ${ }^{1}$ and G. Zimbardo ${ }^{1}$
}

\begin{abstract}
The aim of this work is the analysis of the different transport regimes related to the propagation in the solar wind of solar energetic particles (SEPs) generated by impulsive events like solar flares. A numerical implementation of a Lévy random walk for parallel particle transport is developed, which allows obtaining superdiffusive transport. The comparison between the flows measured by satellites and fluxes extracted from the numerical simulation, will contribute to a deeper understanding of the mechanisms underlying the presence of superdiffusive regimes of SEP transport in interplanetary medium.
\end{abstract}

\section{Introduction}

We carry out an analysis of different transport regimes related to the propagation of solar energetic particles (SEPs) generated by impulsive events like solar flares (e.g., Reames 1999). The analysis of experimental data (e.g., particle fluxes observed by SOHO and ACE satellites) carried out by Trotta \& Zimbardo (2011) shows that the propagation of protons and electrons can correspond to nondiffusive regimes. The experimental energetic particle fluxes will be compared with the numerical simulation, based on a model derived from the theory of Lévy random walk described below.

\section{Superdiffusion and Lévy random walk}

The characterization of the impulsive events follows criteria proposed by Reames (Reames 1999). In the case of superdiffusive or ballistic transport, the mean square displacement grows as $\left\langle\Delta x^{2}(t)\right\rangle=2 D_{\alpha} t^{\alpha}$ with $1<\alpha \leq 2$, and the propagator in the time asymptotic regime has a power law form (Zumofen \& Klafter 1993), so

\footnotetext{
1 Department of Physics, University of Calabria, Ponte P. Bucci, Cubo 31 C, 87036 Arcavacata di Rende (CS), Italy;

e-mail: etrotta@thematica.it; gaetano.zimbardo@fis.unical.it
} 
that a fit of the observed time profiles allows the determination of the transport regime (Perri \& Zimbardo 2007, 2008, 2009). The transport analysis carried out by Trotta \& Zimbardo (2011a,b) assumes that the impulsive events correspond to an injection of particles localized in space and time, so the transport of particles is described by the shape of the propagator. In the case of superdiffusion the propagator for large distances assumes the form $P\left(x-x^{\prime}, t-t^{\prime}\right) \approx a_{0}\left(t-t^{\prime}\right) /\left(x-x^{\prime}\right)^{\gamma}$ which corresponds to the transport law $\left\langle\Delta x^{2}(t)\right\rangle=2 D_{\alpha} t^{\alpha}$ with $\alpha=4-\gamma$.

The different values of $\gamma$ identify different transport regimes: superdiffusive transport for $2<\gamma<3$, and ballistic transport (e.g. scatter-free) for $\gamma>3$ (Zumofen \& Klafter 1993). Such nondiffusive transport regimes are generated by a non Gaussian random walk called Lévy random walk, and characterized by a jump probability $\Psi(x, t)=A|x|^{-\gamma} \delta(x-v t)$ (Klafter et al. 1987), where $x$ represents the jump length. We point out that the power law tails of the jump probability $\Psi$ and of the propagator $P$ are the distinctive feature of superdiffusive transport.

\section{Numerical simulation}

The numerical simulation is based on the test-particle scheme. The equations of motion in the drift approximation are as follows:

$$
\begin{gathered}
\frac{d \boldsymbol{r}}{d t}=v_{\|} \frac{\boldsymbol{B}}{B}+\frac{\boldsymbol{E} \wedge \boldsymbol{B}}{B^{2}}+\frac{\mu}{q} \frac{\boldsymbol{B} \wedge \nabla \boldsymbol{B}}{B^{2}}+\frac{m v_{\|}^{2}}{q} \frac{\boldsymbol{B} \wedge(\boldsymbol{B} \cdot \nabla) \boldsymbol{B}}{B^{4}} \\
\frac{d v_{\|}}{d t}=-\frac{\mu}{m} \nabla B \cdot \frac{\boldsymbol{B}}{B} .
\end{gathered}
$$

The effects of turbulence in the solar wind cause the spreading of particles. These fluctuations are modeled by random changes in the average speed $v_{\|}$which are actually modelled by means of the jump probability distribution $\Psi(x, t)$. The distribution of free path lengths is based on the theory of the Lévy random walk (e.g., Metzler \& Klafter 2000)

$$
\Psi(x, t)=\left[\begin{array}{l}
C \delta(x-v t), \quad x<x_{0} \\
\frac{C}{x_{0}^{-\gamma}}|x|^{-\gamma} \delta(x-v t), \quad x>x_{0}
\end{array}\right.
$$

where $C$ is a normalization constant, and $x_{0}$ is typical length beyond which the power law form of $\Psi$ applies, and which is also used as a normalization length. Further, the delta function $\delta(x-v t)$ enforces the conservation of energy and the space-time coupling. A random number generation algorithm allows having a distribution of jump lengths described by Equation (3.2), based on the relation $\Psi(x) d x=d \xi$ with $\xi$ a random number evenly distributed between 0 and 1 . The corresponding mean square displacement obtained from the simulation is displayed as a function of time in Figure 1 for two values of the power law exponent $\gamma$. Other runs have been done and the results are displayed in Table 1. From Figure 1, it 

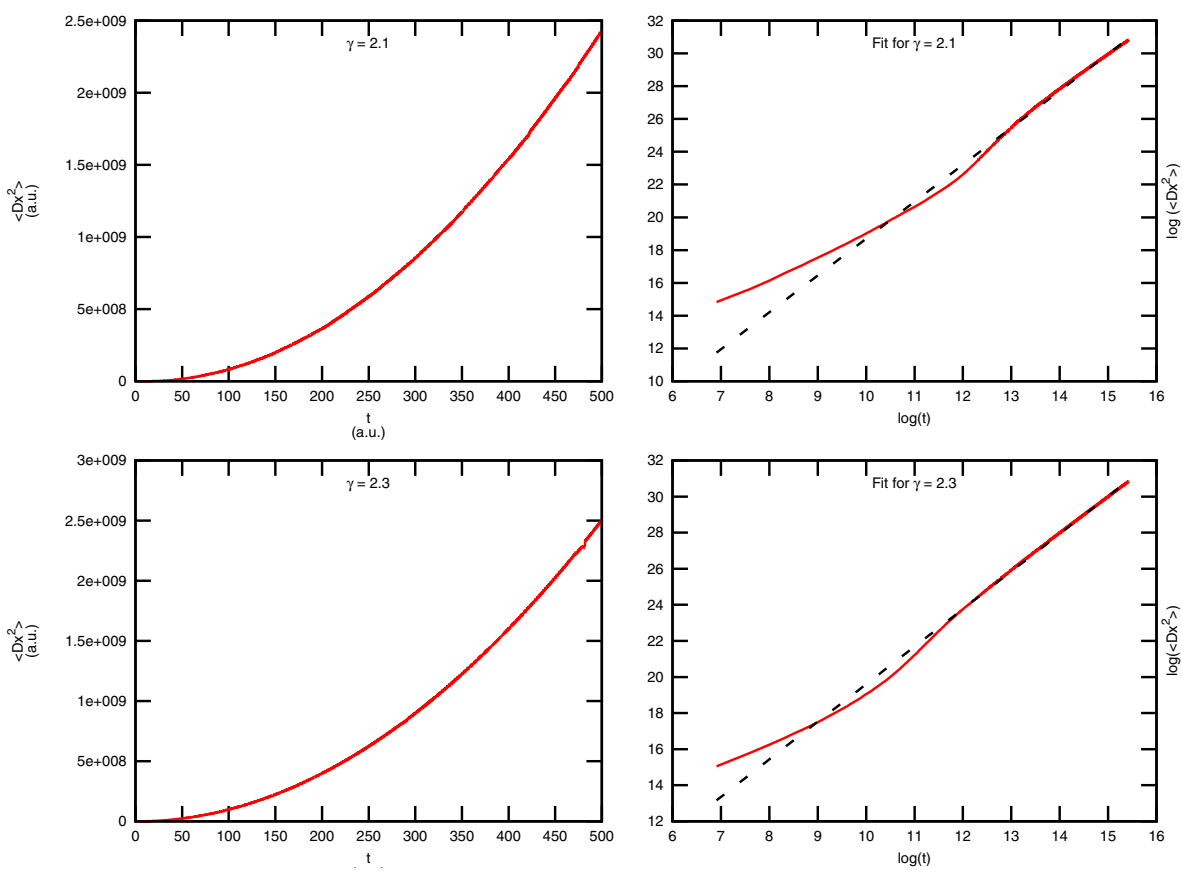

Fig. 1. Simulation results with different values of $\gamma$ for the mean square deviation < $\Delta x^{2}>$ ( in arbitrary units) as a function of time (in arbitrary units), both in linear axes (left) and in log-log axes (right). The dashed lines represent the linear fit to evaluate the diffusive exponent $\alpha$. Upper figures are for $\gamma=2.1$, lower figures for $\gamma=2.3$.

can be seen that the mean square deviation grows faster than linearly with time $(\alpha>1)$, and Table 1 shows that the larger $\gamma$, the smaller $\alpha$. Although this trend is similar to that predicted by the theoretical relation $\alpha=4-\gamma$ (Klafter et al. 1987; Zumofen \& Klafter 1993), a quantitative agreement has not yet been obtained, indicating that further numerical studies are needed.

Table 1. Main results of Lévy random walk simulation. The value of $D_{\alpha}$ and $\alpha$ are obtained from the fit of the mean square deviation while $\gamma$ is the main parameter which is changed in the simulation, see text.

\begin{tabular}{ccc}
\hline \hline$\gamma$ & $\alpha$ & $D_{\alpha}$ \\
\hline \hline 2.1 & 2.0153 & 0.3823 \\
2.3 & 1.9673 & 0.8104 \\
2.5 & 1.8852 & 2.2320 \\
2.7 & 1.0800 & 5.5671 \\
2.9 & 1.7454 & 7.5994 \\
\hline \hline
\end{tabular}




\section{Conclusions}

The aim of the work is to compare the experimental energetic particle fluxes with numerical simulations in order to investigate different transport regimes of energetic particles (in particular, superdiffusive transport which may correspond to the scatter free events reported, e.g., by Krucker \& Lin 2000). These anomalous regimes are important for the mechanism of energetic particle acceleration and for space weather predictions. Here we present a first set of numerical simulations which show how nondiffusive motion can be generated by probability distribution of jump lengths in power law form. In future works, this probability distribution will be implemented into Equation (3.1) to simulate the propagation of energetic particles in the heliosphere.

\section{References}

Klafter, J., Blumen, A., \& Shlesinger, M.F., 1987, Phys. Rev. A, 3081, 35

Krucker, S., \& Lin, R.P., 2000, ApJ, 438, L61

Metzler, R., \& Klafter, J., 2000, Phys. Rep., 339, 1

Perri, S., \& Zimbardo, G., 2007, ApJ, 671, L177

Perri, S., \& Zimbardo, G., 2008, J. Geophys. Res., 113, A03107

Perri, S., \& Zimbardo, G., 2009, ApJ, 693, L118

Reames, D.V., 1999, Space Sci. Rev., 90, 413

Trotta, E.M., \& Zimbardo, G., 2011a, A\&A, 530, id. A130

Trotta, E.M., \& Zimbardo, G., 2011b, Proceedings of IAU Symposium, 274, 198

Zumofen, G., \& Klafter, J., 1993, Phys. Rev. E, 47, 2 